\title{
Controllability, Reachability, and Stabilizability of Finite Automata: A Controllability Matrix Method
}

\author{
Yalu Li, ${ }^{1}$ Wenhui Dou, ${ }^{1}$ Haitao Li $\mathbb{D}^{1,2}$ and Xin Liu ${ }^{1}$ \\ ${ }^{1}$ School of Mathematics and Statistics, Shandong Normal University, Jinan 250014, China \\ ${ }^{2}$ Institute of Data Science and Technology, Shandong Normal University, Jinan 250014, China \\ Correspondence should be addressed to Haitao Li; haitaoli09@gmail.com
}

Received 15 November 2017; Revised 6 January 2018; Accepted 18 January 2018; Published 28 February 2018

Academic Editor: Alessandro Lo Schiavo

Copyright (c) 2018 Yalu Li et al. This is an open access article distributed under the Creative Commons Attribution License, which permits unrestricted use, distribution, and reproduction in any medium, provided the original work is properly cited.

\begin{abstract}
This paper investigates the controllability, reachability, and stabilizability of finite automata by using the semitensor product of matrices. Firstly, by expressing the states, inputs, and outputs as vector forms, an algebraic form is obtained for finite automata. Secondly, based on the algebraic form, a controllability matrix is constructed for finite automata. Thirdly, some necessary and sufficient conditions are presented for the controllability, reachability, and stabilizability of finite automata by using the controllability matrix. Finally, an illustrative example is given to support the obtained new results.
\end{abstract}

\section{Introduction}

In the research field of theoretical computer science, finite automaton is one of the simplest models of computation. Finite automaton is a device whose states take values from a finite set. It receives a discrete sequence of inputs from the outside world and changes its state according to the inputs. The study of finite automata has received many scholars' research interest in the last century [1-5] due to its wide applications in engineering, computer science, and so on.

As we all know, controllability and stabilizability analysis of finite automata are fundamental topics, which are important and necessary to the solvability of many related problems $[1,4,6]$. The concepts of controllability, reachability, and stabilizability of finite automata were defined in [2] by resorting to the classic control theory. The controllability of a deterministic Rabin automaton was studied in [7] by defining the "controllability subset." Kobayashi et al. [8] investigated the state feedback stabilization of a deterministic finite automaton and presented some new results.

Recently, a new matrix product, namely, the semitensor product (STP) of matrices, has been proposed by Cheng et al. [9]. Up to now, STP has been successfully applied to many research fields related to finite-valued systems like Boolean networks [10-20], multivalued logical networks [21-23], game theory $[24,25]$, finite automata $[5,26]$, and so on [27-35]. The main feature of STP is to convert a finite-valued system into an equivalent algebraic form [22]. Thus, STP provides a convenient way for the construction and analysis of finite automata $[5,26]$. Xu and Hong [5] provided a matrixbased algebraic approach for the reachability analysis of finite automata with the help of STP. Yan et al. [26] studied the controllability and stabilizability analysis of finite automata based on STP and presented some novel results. It should be pointed out that although the concepts of controllability, reachability, and stabilizability of finite automata come from classic control theory, there exist fewer results on the construction of controllability matrix for finite automata.

In this paper, we investigate the controllability, reachability, and stabilizability of deterministic finite automata by using STP. The main contribution of this paper is to construct a controllability matrix for finite automata based on the algebraic form. Using the controllability matrix, we present some necessary and sufficient conditions for the controllability, reachability, and stabilizability of finite automata. Compared with the existing results $[5,26]$, our results are more easily verified via MATLAB.

The rest of this paper is organized as follows. Section 2 contains some necessary preliminaries on the semitensor product of matrices and finite automata. Section 3 studies 
the controllability, reachability, and stabilizability of finite automata and presents the main results of this paper. In Section 4, an illustrative example is given to support our new results, which is followed by a brief conclusion in Section 5 .

Notations. $\mathbb{R}, \mathbb{N}$, and $\mathbb{Z}_{+}$denote the set of real numbers, the set of natural numbers, and the set of positive integers, respectively. $\Delta_{n}:=\left\{\delta_{n}^{k}: k=1, \ldots, n\right\}$, where $\delta_{n}^{k}$ denotes the $k$ th column of the $n \times n$ identity matrix $I_{n}$. An $n \times t$ matrix $M$ is called a logical matrix, if $M=\left[\begin{array}{llll}\delta_{n}^{i_{1}} & \delta_{n}^{i_{2}} & \cdots & \delta_{n}^{i_{t}}\end{array}\right]$, which is briefly denoted by $M=\delta_{n}\left[\begin{array}{llll}i_{1} & i_{2} & \cdots & i_{t}\end{array}\right]$. The set of $n \times t$ logical matrices is denoted by $\mathscr{L}_{n \times t}$. Given a real matrix $A$, $\operatorname{Col}_{i}(A), \operatorname{Row}_{j}(A)$, and $(A)_{i, j}$ denote the $i$ th column, the $j$ th row, and the $(i, j)$ th element of $A$, respectively. $A>0$ if and

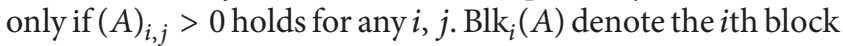
of an $n \times m n$ matrix $A$.

\section{Preliminaries}

2.1. Semitensor Product of Matrices. In this part, we recall some necessary preliminaries on STP. For details, please refer to [9].

Definition 1. Given two matrices $A \in \mathbb{R}^{m \times n}$ and $B \in \mathbb{R}^{p \times q}$, the semitensor product of $A$ and $B$ is defined as

$$
A \ltimes B=\left(A \otimes I_{\alpha / n}\right)\left(B \otimes I_{\alpha / p}\right),
$$

where $\alpha=\operatorname{lcm}(n, p)$ is the least common multiple of $n$ and $p$ and $\otimes$ is the Kronecker product of matrices.

Lemma 2. STP has the following properties:

(1) Let $X \in \mathbb{R}^{t \times 1}$ be a column vector and $A \in \mathbb{R}^{m \times n}$. Then

$$
X \ltimes A=\left(I_{t} \otimes A\right) \ltimes X .
$$

(2) Let $X \in \mathbb{R}^{m \times 1}$ and $Y \in \mathbb{R}^{n \times 1}$ be two column vectors. Then

$$
Y \ltimes X=W_{[m, n]} \ltimes X \ltimes Y,
$$

where $W_{[m, n]} \in \mathscr{L}_{m n \times m n}$ is called the swap matrix.

2.2. Finite Automata. In this subsection, we recall some definitions of finite automata.

A finite automaton is a seven-tuple $A=(X, U, Y, f$, $\left.g, x_{0}, X_{m}\right)$, in which $X, U$, and $Y$ are finite sets of states, input symbols, and outputs, respectively; $x_{0}$ and $X_{m} \subset X$ are the initial state and the set of accepted states; $f$ and $g$ are transition and output functions, which are defined as $f$ : $X \times U \rightarrow 2^{X}$ and $g: X \times U \rightarrow 2^{Y}$, where $2^{X}$ and $2^{Y}$ denote the power set of $X$ and $Y$, respectively; that is, $f(x, u) \subset X$, $g(x, u) \subset Y . U^{*}$ represents the finite string set on $U$, which does not include the empty transition. Given an initial state $x_{0} \in X$ and an input symbol $u \in U$, the function $f$ uniquely determines the next subset of states, that is, $f\left(x_{0}, u\right) \subset X$, while the function $g$ uniquely determines the next subset of outputs; that is, $g\left(x_{0}, u\right) \subset Y$.
Throughout this paper, we only consider the deterministic finite automata; that is, $|f(x, u)| \leq 1$ holds for any $x \in X$ and $u \in U$. In addition, we only investigate the controllability, reachability, and stabilizability of deterministic finite automata, and thus we do not use $Y$ and $g$ in the seven-tuple $A=\left(X, U, Y, f, g, x_{0}, X_{m}\right)$.

In the following, we recall the definitions of controllability, reachability, and stabilizability for deterministic finite automata.

Definition 3. (i) A state $x_{p} \in X$ is said to be controllable to $x_{q} \in X$, if there exists a control sequence $u^{t} \in U^{*}$ such that $f\left(x_{p}, u^{t}\right)=x_{q}$.

(ii) A state $x_{p} \in X$ is said to be controllable, if $x_{p} \in X$ is controllable to any state $x_{q} \in X$.

Definition 4. (i) A state $x_{q} \in X$ is said to be reachable from $x_{p} \in X$, if there exists a control sequence $u^{t} \in U^{*}$ such that $f\left(x_{p}, u^{t}\right)=x_{q}$.

(ii) A state $x_{q} \in X$ is said to be reachable, if $x_{q} \subset X$ is reachable from any state $x_{p} \in X$.

Given two nonempty sets $X_{1} \subseteq X$ and $X_{2} \subseteq X$ satisfying $X_{1} \cup X_{2}=X$ and $X_{1} \cap X_{2}=\emptyset$, we have the following definitions.

Definition 5. A nonempty set of state $X_{1} \subseteq X$ is said to be controllable, if, for any state $x_{q} \in X_{2}$, there exist an $x_{p} \in X_{1}$ and a control sequence $u^{t} \in U^{*}$ such that $f\left(x_{p}, u^{t}\right)=x_{q}$.

Definition 6. A nonempty set of state $X_{2} \subseteq X$ is said to be reachable, if, for any state $x_{p} \in X_{1}$, there exist an $x_{q} \in X_{2}$ and a control sequence $u^{t} \in U^{*}$ such that $f\left(x_{p}, u^{t}\right)=x_{q}$.

Definition 7. A nonempty set of state $X_{1} \subseteq X$ is said to be 1step returnable, if, for any state $x_{0}=x_{p} \in X_{1}$, there exists an input $u_{i} \in U$ such that $f\left(x_{p}, u_{i}\right)=x_{s} \in X_{1}$.

Definition 8. A nonempty set of state $X_{1} \subseteq X$ is said to be stabilizable, if $X_{1}$ is reachable and 1-step returnable.

\section{Main Results}

In this section, we investigate the controllability, reachability, and stabilizability of deterministic finite automata by constructing a controllability matrix.

3.1. Controllability Matrix. For a deterministic finite automaton $A=\left(X, U, Y, f, g, x_{0}, X_{m}\right)$, where $X=\left\{x_{1}, \ldots, x_{n}\right\}$ and $U=\left\{u_{1}, \ldots, u_{m}\right\}$, we identify $x_{i}$ as $\delta_{n}^{i}(i=1, \ldots, n)$ and call $\delta_{n}^{i}$ the vector form of $x_{i}$. Then, $X$ can be denoted as $\Delta_{n}$; that is, $X=\left\{\delta_{n}^{1}, \ldots, \delta_{n}^{n}\right\}$. Similarly, for $U$, we identify $u_{j}$ with $\delta_{m}^{j}(j=1, \ldots, m)$ and call $\delta_{m}^{j}$ the vector form of $u_{j}$. Then, $U=\left\{\delta_{m}^{1}, \ldots, \delta_{m}^{m}\right\}$.

Using the vector form of elements in $X$ and $U$, Yan et al. [26] construct the transition structure matrix (TSM) of $A=$ $\left(X, U, Y, f, g, x_{0}, X_{m}\right)$ as $F=\left[\begin{array}{lll}F_{1} & \cdots & F_{m}\end{array}\right]$. One can see that if 
there exists a control $u_{j} \in U$ which moves state $x_{p}$ to state $x_{q}$, then

$$
\delta_{n}^{q}=F \ltimes \delta_{m}^{j} \ltimes \delta_{n}^{p} .
$$

In this case, $\left(F_{j}\right)_{q, p}=1$. Otherwise, $\left(F_{j}\right)_{q, p}=0$. Thus, setting

$$
M=\sum_{j=1}^{m} F_{j} \in \mathbb{R}^{n \times n}
$$

then one can use $M$ to judge whether or not state $x_{p}$ is controllable to state $x_{q}$ in one step. Precisely, state $x_{p}$ is controllable to state $x_{q}$ in one step, if and only if $(M)_{q, p}>0$.

Now, we show that, for any $t \in \mathbb{Z}_{+}$, state $x_{p}$ is controllable to state $x_{q}$ at the $t$ th step, if and only if $\left(M^{t}\right)_{q, p}>0$. We prove it by induction. Obviously, when $t=1$, the conclusion holds. Assume that the conclusion holds for some $t \in \mathbb{Z}_{+}$. Then, for the case of $t+1$, state $x_{p}$ is controllable to state $x_{q}$ at the $(t+1)$ th step, if and only if there exists some state $x_{r} \in X$ such that state $x_{p}$ is controllable to state $x_{r}$ at the $t$ th step and state $x_{r}$ is controllable to state $x_{q}$ in one step. Hence,

$$
\left(M^{t+1}\right)_{q, p}=\sum_{i=1}^{n}(M)_{q, i}\left(M^{t}\right)_{i, p} \geq(M)_{q, r}\left(M^{t}\right)_{r, p}>0 .
$$

By induction, for any $t \in \mathbb{Z}_{+}$, state $x_{p}$ is controllable to state $x_{q}$ at the th step, if and only if $\left(M^{t}\right)_{q, p}>0$. Thus, $\sum_{t=1}^{\infty} M^{t}$ contains all the controllability information of the finite automata. Noticing that $M$ is an $n \times n$ square matrix, by Cayley-Hamilton theorem, we only need to consider $t \leq n$. Then, we define the controllability matrix for finite automata as follows.

Definition 9. Set $M=\sum_{j=1}^{m} F_{j} \in \mathbb{R}^{n \times n}$. The controllability matrix of finite automata is $C=\sum_{t=1}^{n} M^{t}$. result.

Based on the controllability matrix, we have the following

Algorithm 10. Consider the finite automata $A=(X, U, Y$, $\left.f, g, x_{0}, X_{m}\right)$. Then, the controls which force $x_{p}$ to $x_{q}$ in the shortest time $l$ can be designed by the following steps:

(1) Find the smallest integer $l$ such that, for

$$
\begin{aligned}
\mathscr{F}_{0}^{l} & :=M^{l-1} F \\
& =\left[\begin{array}{lllll}
\operatorname{Blk}_{1}\left(\mathscr{F}_{0}^{l}\right) & \operatorname{Blk}_{2}\left(\mathscr{F}_{0}^{l}\right) & \ldots & \mathrm{Blk}_{m}\left(\mathscr{F}_{0}^{l}\right)
\end{array}\right],
\end{aligned}
$$

there exists a block, say, $\operatorname{Blk}_{\theta}\left(\mathscr{F}_{0}^{l}\right)$, satisfying $\left[\operatorname{Blk}_{\theta}\left(\mathscr{F}_{0}^{l}\right)\right]_{q, p}>0$.

(2) Set $u(0)=\delta_{m}^{\theta}$ and $x_{q}=\delta_{n}^{q}$. If $l=1$, stop. Otherwise, go to Step (3).

(3) Find $r$ and $\eta$ such that $\left[\operatorname{Blk}_{\eta}\left(\mathscr{F}_{0}^{1}\right)\right]_{q, r}>0$ and $\left[\operatorname{Blk}_{\theta}\left(\mathscr{F}_{0}^{l-1}\right)\right]_{r, p}>0$, where $\mathscr{F}_{0}^{1}=F$ and $\mathscr{F}_{0}^{l-1}=$ $M^{l-2} F$. Set $u(l-1)=\delta_{m}^{\eta}$ and $x(l-1)=\delta_{n}^{r}$.

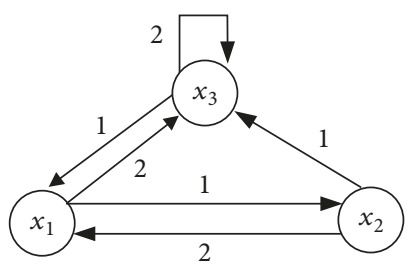

FIgURE 1: A finite automata.

(4) If $l-1=1$, stop. Otherwise, replace $l$ and $q$ by $l-1$ and $r$, respectively, and go to Step (3).

Example 11. Consider a finite automaton $A=(X, U, Y, f$, $\left.g, x_{0}, X_{m}\right)$ given in Figure 1 , where $X=\left\{x_{1}, x_{2}, x_{3}\right\}, U=$ $\{1,2\}$. Suppose that $x_{0}=x_{1}$ and $X_{m}=\left\{x_{3}\right\}$. Then, $X$ can be denoted as $\Delta_{3}=\left\{\delta_{3}^{1}, \delta_{3}^{2}, \delta_{3}^{3}\right\}$. Similarly, $U=\Delta_{2}=\left\{\delta_{2}^{1}, \delta_{2}^{2}\right\}$, $x_{0}=\delta_{3}^{3}, X_{m}=\left\{\delta_{3}^{2}\right\}$.

The transition structure matrix of the finite automata $A$ is

$$
F=\delta_{3}\left[\begin{array}{llllll}
2 & 3 & 1 & 3 & 1 & 3
\end{array}\right] .
$$

Split $F=\left[\begin{array}{ll}F_{1} & F_{2}\end{array}\right]$, where $F_{1}=\delta_{3}\left[\begin{array}{lll}2 & 3 & 1\end{array}\right]$ and $F_{2}=$ $\delta_{3}\left[\begin{array}{lll}3 & 1 & 3\end{array}\right]$. Then,

$$
M=\left[\begin{array}{lll}
0 & 1 & 1 \\
1 & 0 & 0 \\
1 & 1 & 1
\end{array}\right] .
$$

Thus, the controllability matrix is

$$
C=\sum_{t=1}^{3} M^{t}=\left[\begin{array}{lll}
4 & 5 & 5 \\
3 & 2 & 2 \\
7 & 7 & 7
\end{array}\right] .
$$

By Algorithm 10, one can obtain that $\left[\operatorname{Blk}_{1}\left(\mathscr{F}_{0}^{2}\right)\right]_{2,3}>0$. Setting $x_{p}=\delta_{3}^{3}, u(0)=\delta_{2}^{1}$, and $x_{q}=\delta_{3}^{2}$, one can find $r=1$ and $\eta=1$ such that $\left[\operatorname{Blk}_{1}\left(\mathscr{F}_{0}^{1}\right)\right]_{2,1}>0$ and $\left[\operatorname{Blk}_{1}\left(\mathscr{F}_{0}^{1}\right)\right]_{1,3}>0$. Let $u(1)=\delta_{2}^{1}$ and $x_{1}=\delta_{3}^{1}$. Hence, state $x_{3}$ is controllable to state $x_{2}$ at the 2 nd step.

3.2. Controllability, Reachability, and Stabilizability. In this part, we study the controllability, reachability, and stabilizability of deterministic finite automata based on the controllability matrix.

According to the meaning of controllability matrix, we have the following results.

Theorem 12. The state $x_{p} \in X$ is controllable, if and only if

$$
\mathrm{Col}_{p}(C)>0 \text {. }
$$

Proof.

Necessity. Suppose that the state $x_{p} \in X$ is controllable. By Definition 3, $x_{p} \in X$ is controllable to any state $x_{q} \in X$. Based on (4), one can see that there exists a control sequence $\left\{u_{i_{\alpha}}: \alpha=0, \ldots, s-1\right\} \subseteq U(1 \leq s \leq n)$ satisfying 
$\delta_{n}^{q}=F_{i_{s-1}} \cdots F_{i_{0}} \ltimes \delta_{n}^{p}$. Thus, $\left(F_{i_{s-1}} \cdots F_{i_{0}}\right)_{q, p}>0$, which implies that

$$
(C)_{q, p} \geq\left(F_{i_{s-1}} \cdots F_{i_{0}}\right)_{q, p}>0 \text {. }
$$

From the arbitrariness of $q$, we have $\operatorname{Col}_{p}(C)>0$.

Sufficiency. Suppose that $\operatorname{Col}_{p}(C)>0$ holds. Then, for any state $x_{q} \in X$, one can find some $\left(F_{i_{s-1}} \cdots F_{i_{0}}\right)_{q, p}>0$. Therefore, under the control sequence $\left\{u_{i_{\alpha}}: \alpha=0, \ldots, s-1\right\} \subseteq U$, the state $x_{p} \subset X$ is controllable to $x_{q} \in X$. From the arbitrariness of $q$, the state $x_{p} \in X$ is controllable.

Theorem 13. The state $x_{q} \in X$ is reachable, if and only if

$$
\operatorname{Row}_{q}(C)>0 \text {. }
$$

Proof.

Necessity. Suppose that the state $x_{q} \in X$ is reachable. By Definition $4, x_{q} \in X$ is reachable to any state $x_{p} \in X$. One can obtain from (4) that there exists a control sequence $\left\{u_{i_{\alpha}}: \alpha=\right.$ $0, \ldots, s-1\} \subseteq U(1 \leq s \leq n)$ satisfying $\delta_{n}^{q}=F_{i_{s-1}} \cdots F_{i_{0}} \ltimes \delta_{n}^{p}$. Thus, $\left(F_{i_{s-1}} \cdots F_{i_{0}}\right)_{q, p}>0$, which shows that

$$
(C)_{q, p} \geq\left(F_{i_{s-1}} \cdots F_{i_{0}}\right)_{q, p}>0 \text {. }
$$

From the arbitrariness of $p$, one can conclude that $\operatorname{Row}_{q}(C)>$ 0 .

Sufficiency. Suppose that $\operatorname{Row}_{q}(C)>0$. Then, for any state $x_{p} \in X$, there exists some $\left(F_{i_{s-1}} \cdots F_{i_{0}}\right)_{q, p}>0$. Hence, under the control sequence $\left\{u_{i_{\alpha}}: \alpha=0, \ldots, s-1\right\} \subseteq U$, the state $x_{q} \subset X$ is reachable to $x_{p} \in X$. By Definition 4 , the state $x_{q} \in X$ is reachable.

Given two nonempty sets $X_{1}=\left\{\delta_{n}^{p_{1}}, \ldots, \delta_{n}^{p_{s}}\right\}$ and $X_{2}=$ $\left\{\delta_{n}^{p_{s+1}}, \ldots, \delta_{n}^{p_{n}}\right\}$, where $X_{1} \cup X_{2}=X$ and $X_{1} \cap X_{2}=\emptyset$, define

$$
\begin{aligned}
& C_{X_{1}}=\left[\sum_{\beta=1}^{s}(C)_{p_{s+1}, p_{\beta}} \cdots \sum_{\beta=1}^{s}(C)_{p_{n}, p_{\beta}}\right] \text {, } \\
& R_{X_{2}}=\left[\sum_{\gamma=s+1}^{n}(C)_{p_{\gamma}, p_{1}} \cdots \sum_{\gamma=s+1}^{n}(C)_{p_{\gamma}, p_{s}}\right] \text {. }
\end{aligned}
$$

Based on Theorems 12 and 13, we have the following result.

Theorem 14. (i) The nonempty set $X_{1} \subseteq X$ is controllable, if and only if $C_{X_{1}}>0$.

(ii) The nonempty set $X_{2} \subseteq X$ is reachable, if and only if $R_{X_{2}}>0$.

\section{Proof.}

(i) Necessity. Suppose that the nonempty set $X_{1} \subseteq X$ is controllable. By Definition 5, for any state $\delta_{n}^{p_{\gamma}} \in X_{2}$, there exist a $\delta_{n}^{p_{\beta}} \in X_{1}$ and a control sequence $u^{t} \in U^{*}$ such that $f\left(\delta_{n}^{p_{\beta}}, u^{t}\right)=\delta_{n}^{p_{\gamma}}$. Based on Theorems 12 and 13, for a fixed $\delta_{n}^{p_{\gamma}} \in X_{2}, \gamma=s+1, s+2, \ldots, n$, at least one of the following cases is true: $(C)_{p_{\gamma}, p_{1}}>0,(C)_{p_{\gamma}, p_{2}}>0, \ldots,(C)_{p_{\gamma}, p_{s}}>0$. Therefore, for a fixed $\delta_{n}^{p_{\gamma}} \in X_{2}$, one can conclude that $\sum_{\beta=1}^{s}(C)_{p_{\gamma}, p_{\beta}}>0$. From the arbitrariness of $p_{\gamma}$, one can see that

$$
C_{X_{1}}=\left[\sum_{\beta=1}^{s}(C)_{p_{s+1}, p_{\beta}} \cdots \sum_{\beta=1}^{s}(C)_{p_{n}, p_{\beta}}\right]>0 .
$$

Sufficiency. Suppose that $C_{X_{1}}=\left[\sum_{\beta=1}^{s}(C)_{p_{s+1}, p_{\beta}} \ldots\right.$ $\left.\sum_{\beta=1}^{s}(C)_{p_{n}, p_{\beta}}\right]>0$. Then, for any $\gamma=s+1, \ldots, n$, we have $\sum_{\beta=1}^{s}(C)_{p_{\gamma}, p_{\beta}}>0$. It means that, for any state $\delta_{n}^{p_{\gamma}} \in X_{2}$, there exist a $\delta_{n}^{p_{\beta}} \in X_{1}$ and a control sequence $u^{t} \in U^{*}$ such that $f\left(\delta_{n}^{p_{\beta}}, u^{t}\right)=\delta_{n}^{p_{\gamma}}$. By Definition 5, the nonempty set $X_{1} \subseteq X$ is controllable.

(ii) Necessity. Suppose that the nonempty set $X_{2} \subseteq X$ is reachable. By Definition 6 , for any state $\delta_{n}^{p_{\beta}} \in X_{1}$, there exist a $\delta_{n}^{p_{\gamma}} \in X_{2}$ and a control sequence $u^{t} \in U^{*}$ such that $f\left(\delta_{n}^{p_{\beta}}, u^{t}\right)=\delta_{n}^{p_{\gamma}}$. Based on Theorems 12 and 13, for a fixed $\delta_{n}^{p_{\beta}} \in X_{1}, \beta=1,2, \ldots, s$, at least one of the following cases is true: $(C)_{p_{s+1}, p_{\beta}}>0,(C)_{p_{s+2}, p_{\beta}}>0, \ldots,(C)_{p_{n}, p_{\beta}}>0$. Therefore, for a fixed $\delta_{n}^{p_{\beta}} \in X_{1}$, one can see that $\sum_{\gamma=s+1}^{n}(C)_{p_{\gamma}, p_{\beta}}>0$. From the arbitrariness of $p_{\beta}$, we have

$$
\left[\sum_{\gamma=s+1}^{n}(C)_{p_{\gamma}, p_{1}} \cdots \sum_{\gamma=s+1}^{n}(C)_{p_{\gamma}, p_{s}}\right]>0 .
$$

Sufficiency. Suppose that $\left[\sum_{\gamma=s+1}^{n}(C)_{p_{\gamma}, p_{1}} \cdots \sum_{\gamma=s+1}^{n}(C)_{p_{\gamma}, p_{s}}\right]$ $>0$. Then, for any $\beta=1,2, \ldots, s$, we have $\sum_{\gamma=s+1}^{n}(C)_{p_{\gamma}, p_{\beta}}>0$. It means that, for any state $\delta_{n}^{p_{\beta}} \in X_{1}$, there exist a $\delta_{n}^{p_{\gamma}} \in X_{2}$ and a control sequence $u^{t} \in U^{*}$ such that $f\left(\delta_{n}^{p_{\beta}}, u^{t}\right)=\delta_{n}^{p_{\gamma}}$. By Definition 6, the nonempty set $X_{2} \subseteq X$ is reachable.

Finally, we study the stabilizability of deterministic finite automata.

For $X_{1}=\left\{\delta_{n}^{p_{1}}, \ldots, \delta_{n}^{p_{s}}\right\}$ and $X_{2}=\left\{\delta_{n}^{p_{s+1}}, \ldots, \delta_{n}^{p_{n}}\right\}$, define

$$
M_{X_{1}}=\left[\sum_{i=1}^{s}(M)_{p_{i}, p_{1}} \cdots \sum_{i=1}^{s}(M)_{p_{i}, p_{s}}\right] .
$$

Theorem 15. The nonempty set $X_{1} \subseteq X$ is 1-step returnable, if and only if $M_{X_{1}}>0$.

Proof. By Definition 7, one can see that the nonempty set $X_{1} \subseteq X$ is 1-step returnable, if and only if, for any state $\delta_{n}^{p_{\beta}} \in X_{1}$, there exist an input $u_{i} \in U$ and some $\delta_{n}^{p_{i}} \in X_{1}$ such that $f\left(\delta_{n}^{p_{\beta}}, u_{i}\right)=\delta_{n}^{p_{i}}$, that is, for a fixed $\delta_{n}^{p_{\beta}} \in X_{1}, \beta=$ $1,2, \ldots, s$, at least one of the following cases is true: $(M)_{p_{1}, p_{\beta}}>$ $0,(M)_{p_{2}, p_{\beta}}>0, \ldots,(M)_{p_{s}, p_{\beta}}>0$. Hence, $\sum_{i=1}^{s}(M)_{p_{i}, p_{\beta}}>0$, $\forall \beta=1, \ldots, s$. From the arbitrariness of $p_{\beta}$, one can obtain that $M_{X_{1}}>0$. 
Based on Theorems 14 and 15, we have the following result.

Corollary 16. The nonempty set $X_{1} \subseteq X$ is stabilizable, if and only if $M_{X_{1}}>0$ and $R_{X_{1}}>0$.

Proof. By Definition 8, $X_{1} \subseteq X$ is stabilizable, if and only if $X_{1}$ is reachable and 1-step returnable. Based on Theorems 14 and 15 , the conclusion follows.

Remark 17. Compared with the existing results on the controllability and stabilizability of deterministic finite automata $[5,26]$, the main advantage of our results is to propose a unified tool, that is, controllability matrix, for the study of deterministic finite automata. The new conditions are more easily verified via MATLAB.

\section{An Illustrative Example}

Consider the finite automata $A=\left(X, U, Y, f, g, x^{0}, X^{m}\right)$ given in Figure 2, where $X=\left\{x_{1}, x_{2}, x_{3}, x_{4}\right\}$ and $U=\{1,2\}$.

From Figure 2 , we can see that $x_{1} \stackrel{1}{\rightarrow} x_{2} \stackrel{1}{\rightarrow} x_{3} \stackrel{2}{\rightarrow} x_{4}$ and $x_{1} \stackrel{1}{\rightarrow} x_{2} \stackrel{2}{\rightarrow} x_{1}$. Therefore, by Definition 3 , one can obtain that $x_{1}$ is controllable. Similarly, by Definition 3 , we conclude that $x_{2}, x_{3}$, and $x_{4}$ are also controllable. By Definition 4 , we can also find that all the states are reachable.

Assume that $X_{1}=\left\{x_{1}, x_{2}\right\}$ and $X_{2}=\left\{x_{3}, x_{4}\right\}$. Since $x_{1} \in X_{1} \stackrel{1}{\rightarrow} x_{2} \stackrel{1}{\rightarrow} x_{3} \in X_{2}$ and $x_{1} \in X_{1} \stackrel{2}{\rightarrow} x_{4} \in X_{2}$, by Definition 5 , one can see that $X_{1}$ is controllable. Similarly, we also obtain that $X_{2}$ is controllable. Since $x_{3} \stackrel{2}{\rightarrow} x_{4} \stackrel{1}{\rightarrow} x_{2} \stackrel{2}{\rightarrow} x_{1}$ and $x_{1} \stackrel{1}{\rightarrow} x_{2} \stackrel{1}{\rightarrow} x_{3} \stackrel{2}{\rightarrow} x_{4}$, by Definition 6 , we can obtain that $X_{1}$ and $X_{2}$ are reachable. From Figure 2, we can see that $x_{1} \stackrel{1}{\rightarrow} x_{2}, x_{2} \stackrel{2}{\rightarrow} x_{1}, x_{3} \stackrel{2}{\rightarrow} x_{4}$, and $x_{4} \stackrel{2}{\rightarrow} x_{3}$. Hence, by Definition $7, X_{1}$ and $X_{2}$ are 1-step returnable. By Definition 8 , the sets $X_{1}$ and $X_{2}$ are stabilizable.

Now, we check the above properties based on the controllability matrix.

The transition structure matrix of the finite automata $A$ is

$$
F=\delta_{4}\left[\begin{array}{llllllll}
2 & 3 & 3 & 2 & 4 & 1 & 4 & 3
\end{array}\right] .
$$

Split $F=\left[\begin{array}{ll}F_{1} & F_{2}\end{array}\right]$, where $F_{1}=\delta_{4}\left[\begin{array}{llll}2 & 3 & 3 & 2\end{array}\right]$ and $F_{2}=$ $\delta_{4}\left[\begin{array}{llll}4 & 1 & 4 & 3\end{array}\right]$. Then,

$$
M=\left[\begin{array}{llll}
0 & 1 & 0 & 0 \\
1 & 0 & 0 & 1 \\
0 & 1 & 1 & 1 \\
1 & 0 & 1 & 0
\end{array}\right] .
$$

Thus, the controllability matrix is

$$
C=\sum_{t=1}^{4} M^{t}=\left[\begin{array}{cccc}
3 & 4 & 2 & 3 \\
7 & 5 & 5 & 6 \\
12 & 13 & 14 & 14 \\
8 & 8 & 9 & 7
\end{array}\right]
$$

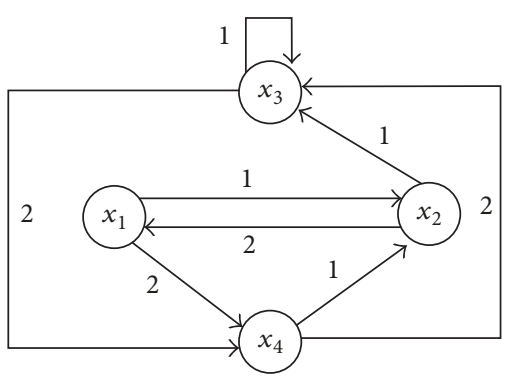

Figure 2: A finite automata.

Since all rows and columns of $C$ are positive, by Theorems 12 and 13 , any state $x_{i} \in X$ is controllable and reachable, $i=$ $1,2,3,4$.

A simple calculation gives $C_{X_{1}}=\left[\begin{array}{ll}25 & 16\end{array}\right], R_{X_{1}}=\left[\begin{array}{ll}7 & 9\end{array}\right]$, $C_{X_{2}}=\left[\begin{array}{ll}5 & 11\end{array}\right], R_{X_{2}}=\left[\begin{array}{ll}20 & 21\end{array}\right], M_{X_{1}}=\left[\begin{array}{ll}10 & 9\end{array}\right]$, and $M_{X_{2}}=$ [23 21 ]. By Theorems 14 and 15 and Corollary 16, $X_{1}$ and $X_{2}$ are controllable, reachable, 1-step returnable, and stabilizable, respectively.

\section{Conclusion}

In this paper, we have investigated the controllability, reachability, and stabilizability of deterministic finite automata by using the semitensor product of matrices. We have obtained the algebraic form of finite automata by expressing the states, inputs, and outputs as vector forms. Based on the algebraic form, we have defined the controllability matrix for deterministic finite automata. In addition, using the controllability matrix, we have presented several necessary and sufficient conditions for the controllability, reachability, and stabilizability of finite automata. The study of an illustrative example has shown that the obtained new results are effective.

\section{Conflicts of Interest}

The authors declare that there are no conflicts of interest regarding the publication of this paper.

\section{Acknowledgments}

The research was supported by the National Natural Science Foundation of China under Grants 61374065 and 61503225, the Natural Science Foundation of Shandong Province under Grant ZR2015FQ003, and the Natural Science Fund for Distinguished Young Scholars of Shandong Province under Grant JQ201613.

\section{References}

[1] S. Abdelwahed and W. M. Wonham, "Blocking Detection in Discrete Event Systems," in Proceedings of the American Control Conference, pp. 1673-1678, USA, 2003.

[2] M. Dogruel and U. Ozguner, "Controllability, reachability, stabilizability and state reduction in automata," in Proceedings of the IEEE International Symposium on Intelligent Control, pp. 192-197, Glasgow, UK, 1992. 
[3] Y. Gang, "Decomposing a kind of weakly invertible finite automata with delay 2," Journal of Computer Science and Technology, vol. 18, no. 3, pp. 354-360, 2003.

[4] J. Lygeros, C. Tomlin, and S. Sastry, "Controllers for reachability specifications for hybrid systems," Automatica, vol. 35, no. 3, pp. 349-370, 1999.

[5] X. Xu and Y. Hong, "Matrix expression and reachability analysis of finite automata," Control Theory and Technology, vol. 10, no. 2, pp. 210-215, 2012.

[6] A. Casagrande, A. Balluchi, L. Benvenuti, A. Policriti, T. Villa, and A. Sangiovanni-Vincentelli, "Improving reachability analysis of hybrid automata for engine control," in Proceedings of the 43rd IEEE Conference on Decision and Control (CDC), pp. 2322-2327, 2004.

[7] J. Thistle and W. Wonham, "Control of infinite behavior of finite automata," SIAM Journal on Control and Optimization, vol. 32, no. 4, pp. 1075-1097, 1994.

[8] K. Kobayashi, J. Imura, and K. Hiraishi, "Stabilization of finite automata with application to hybrid systems control," Discrete Event Dynamic Systems, vol. 21, no. 4, pp. 519-545, 2011.

[9] D. Cheng, H. Qi, and Z. Li, Analysis and Control of Boolean Network: A Semi-Tensor Product Approach, Communications and Control Engineering Series, Springer, London, UK, 2011.

[10] E. Fornasini and M. E. Valcher, "On the periodic trajectories of Boolean control networks," Automatica, vol. 49, no. 5, pp. 15061509, 2013.

[11] Y. Guo, P. Wang, W. Gui, and C. Yang, "Set stability and set stabilization of Boolean control networks based on invariant subsets," Automatica, vol. 61, pp. 106-112, 2015.

[12] D. Laschov and M. Margaliot, "Minimum-time control of Boolean networks," SIAM Journal on Control and Optimization, vol. 51, no. 4, pp. 2869-2892, 2013.

[13] F. Li and J. Sun, "Controllability and optimal control of a temporal Boolean network," Neural Networks, vol. 34, pp. 10-17, 2012.

[14] F. Li, "Pinning control design for the synchronization of two coupled boolean networks," IEEE Transactions on Circuits and Systems II: Express Briefs, vol. 63, no. 3, pp. 309-313, 2016.

[15] H. Li, L. Xie, and Y. Wang, "On robust control invariance of Boolean control networks," Automatica, vol. 68, pp. 392-396, 2016.

[16] H. Li, Y. Wang, and L. Xie, "Output tracking control of Boolean control networks via state feedback: Constant reference signal case," Automatica, vol. 59, article 6422, pp. 54-59, 2015.

[17] H. Li and Y. Wang, "Controllability analysis and control design for switched Boolean networks with state and input constraints," SIAM Journal on Control and Optimization, vol. 53, no. 5, pp. 2955-2979, 2015.

[18] H. Li, L. Xie, and Y. Wang, "Output regulation of Boolean control networks," Institute of Electrical and Electronics Engineers Transactions on Automatic Control, vol. 62, no. 6, pp. 29932998, 2017.

[19] J. Lu, J. Zhong, C. Huang, and J. Cao, "On pinning controllability of Boolean control networks," Institute of Electrical and Electronics Engineers Transactions on Automatic Control, vol. 61, no. 6, pp. 1658-1663, 2016.

[20] M. Meng, L. Liu, and G. Feng, "Stability and $l_{1}$ gain analysis of Boolean networks with Markovian jump parameters," Institute of Electrical and Electronics Engineers Transactions on Automatic Control, vol. 62, no. 8, pp. 4222-4228, 2017.
[21] Z. Liu, Y. Wang, and H. Li, "New approach to derivative calculation of multi-valued logical functions with application to fault detection of digital circuits," IET Control Theory \& Applications, vol. 8, no. 8, pp. 554-560, 2014.

[22] J. Lu, H. Li, Y. Liu, and F. Li, "Survey on semi-tensor product method with its applications in logical networks and other finite-valued systems," IET Control Theory \& Applications, vol. 11, no. 13, pp. 2040-2047, 2017.

[23] Y. Wu and T. Shen, "An algebraic expression of finite horizon optimal control algorithm for stochastic logical dynamical systems," Systems \& Control Letters, vol. 82, article 3915, pp. 108114, 2015.

[24] D. Cheng, F. He, H. Qi, and T. Xu, "Modeling, analysis and control of networked evolutionary games," Institute of Electrical and Electronics Engineers Transactions on Automatic Control, vol. 60, no. 9, pp. 2402-2415, 2015.

[25] P. Guo, H. Zhang, F. E. Alsaadi, and T. Hayat, "Semi-tensor product method to a class of event-triggered control for finite evolutionary networked games," IET Control Theory \& Applications, vol. 11, no. 13, pp. 2140-2145, 2017.

[26] Y. Yan, Z. Chen, and Z. Liu, "Semi-tensor product approach to controllability and stabilizability of finite automata," Journal of Systems Engineering and Electronics, vol. 26, no. 1, pp. 134-141, 2015.

[27] D. Cheng and H. Qi, "Non-regular feedback linearization of nonlinear systems via a normal form algoithm," Automatica, vol. 40, pp. 439-447, 2004.

[28] H. Li, G. Zhao, M. Meng, and J. Feng, "A survey on applications of semi-tensor product method in engineering," Science China Information Sciences, vol. 61, no. 1, Article ID 010202, 2018.

[29] Z. Li, Y. Qiao, H. Qi, and D. Cheng, "Stability of switched polynomial systems," Journal of Systems Science and Complexity, vol. 21, no. 3, pp. 362-377, 2008.

[30] Y. Liu, H. Chen, J. Lu, and B. Wu, "Controllability of probabilistic Boolean control networks based on transition probability matrices," Automatica, vol. 52, pp. 340-345, 2015.

[31] Y. Wang, C. Zhang, and Z. Liu, "A matrix approach to graph maximum stable set and coloring problems with application to multi-agent systems," Automatica, vol. 48, no. 7, pp. 1227-1236, 2012.

[32] Y. Yan, Z. Chen, and Z. Liu, "Solving type-2 fuzzy relation equations via semi-tensor product of matrices," Control Theory and Technology, vol. 12, no. 2, pp. 173-186, 2014.

[33] K. Zhang, L. Zhang, and L. Xie, "Invertibility and nonsingularity of Boolean control networks," Automatica, vol. 60, article 6475, pp. 155-164, 2015.

[34] J. Zhong, J. Lu, Y. Liu, and J. Cao, "Synchronization in an array of output-coupled boolean networks with time delay," IEEE Transactions on Neural Networks and Learning Systems, vol. 25, no. 12, pp. 2288-2294, 2014.

[35] Y. Zou and J. Zhu, "System decomposition with respect to inputs for Boolean control networks," Automatica, vol. 50, no. 4, pp. 1304-1309, 2014. 


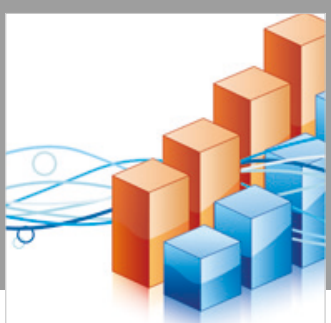

Advances in

Operations Research

\section{-n-m}
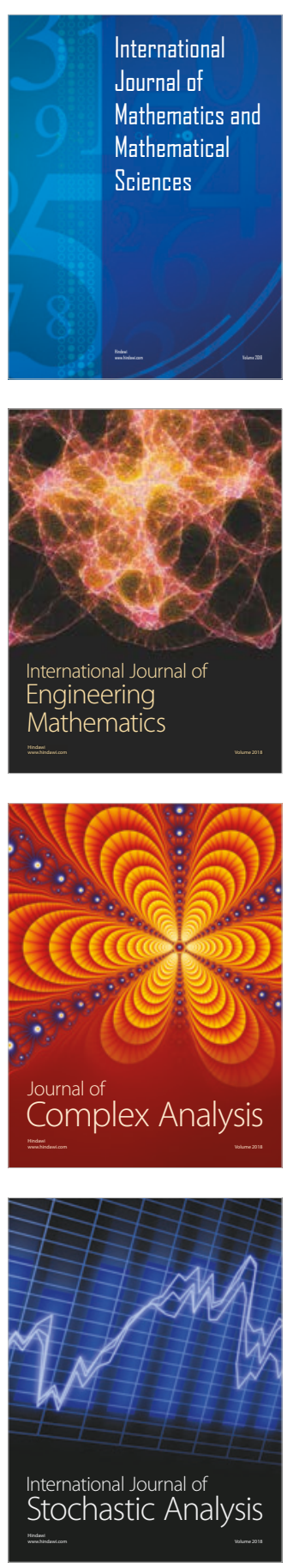
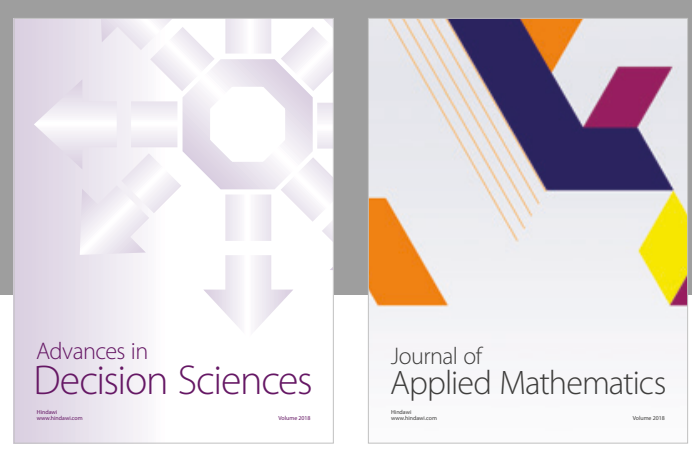

Journal of

Applied Mathematics
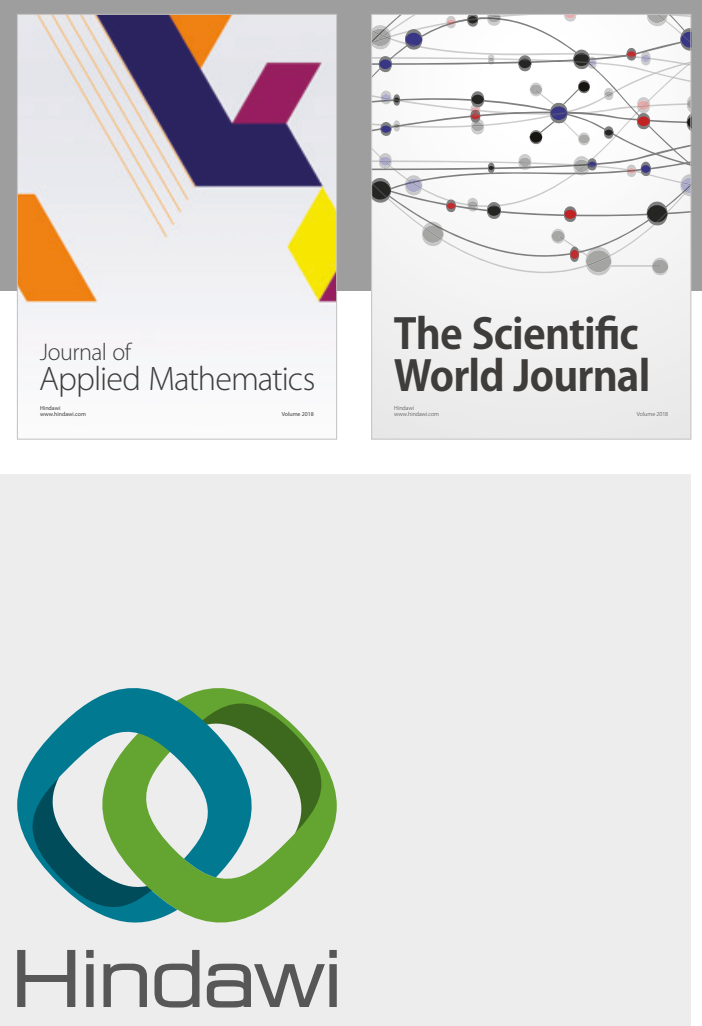

Submit your manuscripts at

www.hindawi.com

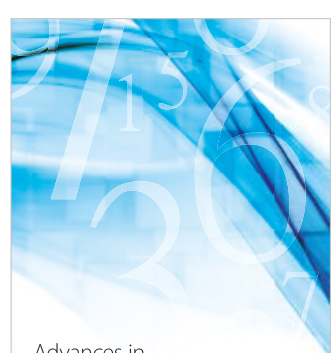

Advances in
Numerical Analysis
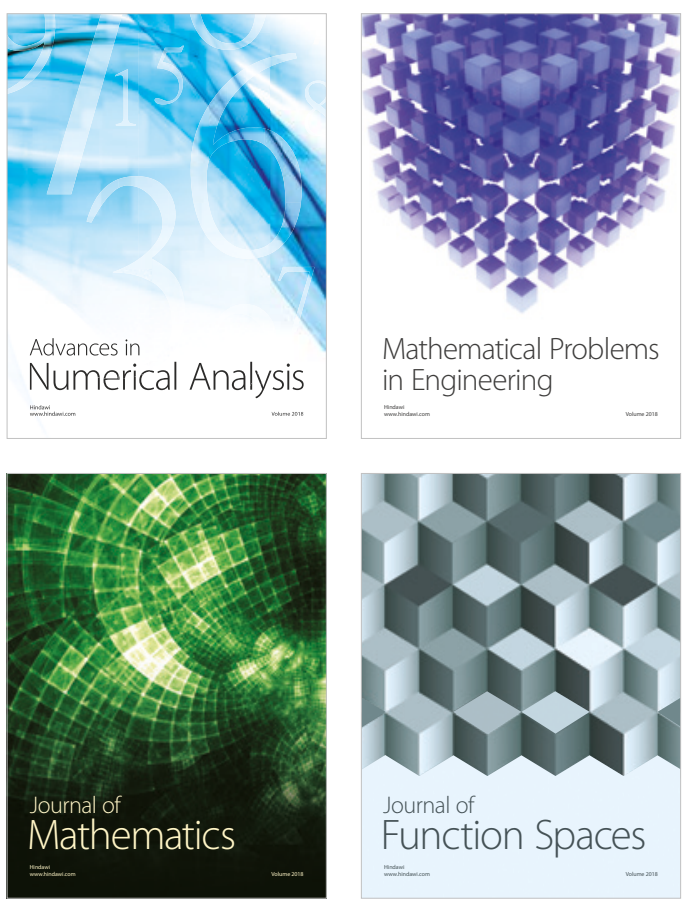

Mathematical Problems in Engineering

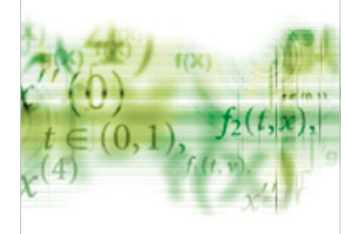

International Journal of

Differential Equations

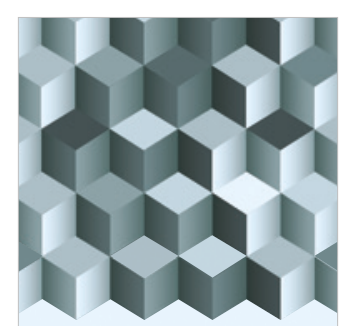

Journal of

Function Spaces

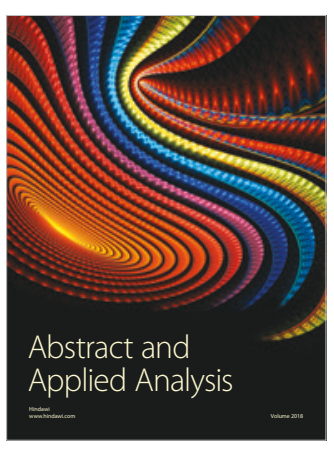

The Scientific

World Journal

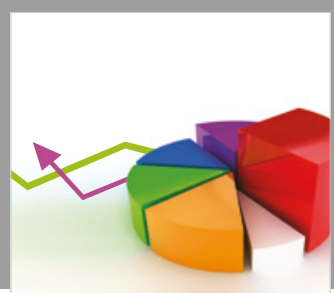

Journal of

Probability and Statistics
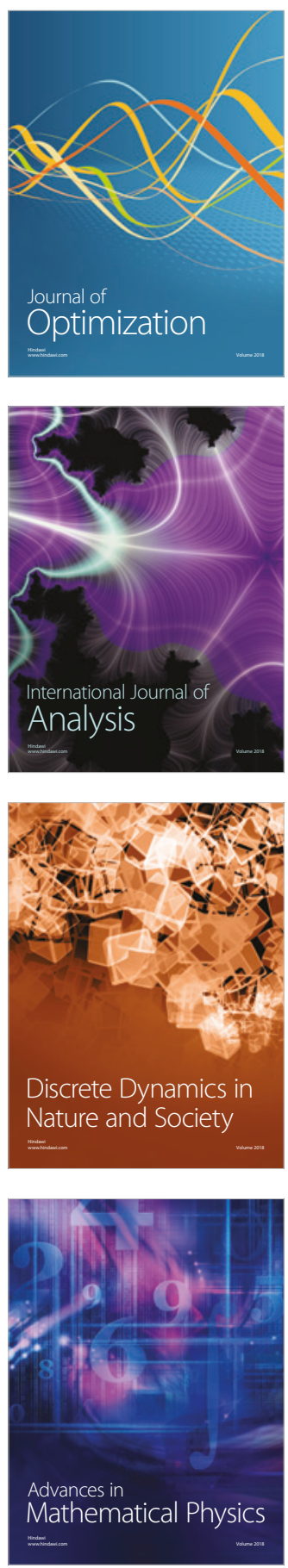